\title{
MASSA ESPECÍFICA E RETRATIBILIDADE DA MADEIRA DE SEIS ESPÉCIES DE EUCALIPTO CULTIVADAS NO LITORAL DE SANTA CATARINA
}

\author{
Jackson Roberto Eleotério $^{1}$, Douglas Reichert ${ }^{2}$, Kerling Fabiane Hornburg ${ }^{3}$, Isalena Meneguelli ${ }^{2}$ \\ ${ }^{1}$ Universidade Regional de Blumenau, Departamento de Engenharia Florestal, Blumenau, Santa Catarina, Brasil - jreleote@ furb.br \\ ${ }^{2}$ Universidade Regional de Blumenau, Curso de Engenharia Florestal, Blumenau, Santa Catarina, Brasil - reichert_gog@gmail.com; \\ isalenameneg@gmail.com \\ ${ }^{3}$ Universidade Regional de Blumenau, Blumenau, Santa Catarina, Brasil - kerlingh@gmail.com
}

Recebido para publicação: 10/12/2013 - Aceito para publicação: 29/09/2014

\begin{abstract}
Resumo
O objetivo deste trabalho é determinar e comparar a massa específica básica e aparente a $12 \%$ de umidade e os coeficientes de contração radial, tangencial e volumétrico de cinco espécies e um híbrido de eucalipto. As amostras de Corymbia torelliana, Eucalyptus cloeziana, Eucalyptus pilularis e Eucalyptus resinifera foram retiradas de árvores com 17 anos; as de Eucalyptus grandis, de árvores com 14 anos; e as de Eucalyptus grandis x Eucalyptus urophylla, de árvores com oito anos, todas plantadas no litoral norte de Santa Catarina. A madeira do híbrido Eucalyptus grandis x Eucalyptus urophylla e a de Eucalyptus grandis e de Eucalyptus pilularis classificam-se como leve, e a das demais espécies é classificada como madeira moderadamente pesada. Independentemente da contração analisada, os grupos formados na comparação de médias foram os mesmos. Os coeficientes de contração radial de Eucalyptus cloeziana e Eucalyptus resinifera são altos; já os coeficientes de contração tangencial e volumétrico de Eucalyptus grandis e volumétrico de Eucalyptus pilularis são baixos; e os demais podem ser classificados como médios. A variação na massa específica não é acompanhada de proporcional variação nos coeficientes de contração, resultando em baixas e moderadas correlações entre essas variáveis, apesar de significativas.
\end{abstract}

Palavras-chave: Propriedades físicas; contração; Eucalyptus; Corymbia.

\begin{abstract}
Specific gravity and shrinkage coefficients of wood of six species of eucalypts cultivated on the coast of Santa Catarina. This work aims at determining and comparing the basic and $12 \%$ moisture content specific gravity and radial, tangential and volumetric shrinkage coefficients of five species and a hybrid of eucalypts. The samples of Corymbia torelliana, Eucalyptus cloeziana, Eucalyptus pilularis, Eucalyptus resinifera were extracted from 17 years old trees; ones of Eucalyptus grandis from 14 years old trees and the ones of Eucalyptus grandis x Eucalyptus urophylla from eight years old trees, all of them planted on the North Coast of Santa Catarina State. The wood of the hybrid Eucalyptus grandis $\mathrm{x}$ Eucalyptus urophylla, of the Eucalyptus grandis and of the Eucalyptus pilularis can be classified as light, the others can be classified as moderately heavy. Independently on the shrinkage analyzed, the groups formed during the multiple mean comparing are the same. The radial shrinkage coefficient of Eucalyptus cloeziana and that of Eucalyptus resinifera are high. However, the tangential and volumetric shrinkage coefficients of Eucalyptus grandis and the volumetric shrinkage coefficient of Eucalyptus pilularis are low; the others can be classified as medium. The variation on specific gravity is not followed by proportional variation on the shrinkage coefficients, resulting in low and moderate correlations between coefficients even tough significant.

Keywords: Physical properties; shrinkage coefficients; Eucalyptus; Corymbia.
\end{abstract}

\section{INTRODUÇÃO}

A utilização da madeira de espécies dos gêneros Eucalyptus e Corymbia na fabricação de produtos de madeira sólida tem sua viabilidade relacionada com o desempenho no processamento e com as propriedades físicas e mecânicas desse material.

FLORESTA, Curitiba, PR, v. 45, n. 2, p. 329 - 336, abr. / jun. 2015

Eleotério, J. R. et al.

ISSN eletrônico 1982-4688 / ISSN impresso 0015-3826

DOI: $10.5380 /$ rf.v45i2.34699 
A massa específica é um dos mais importantes indicadores de qualidade da madeira. Essa propriedade é fortemente correlacionada com as propriedades mecânicas e moderadamente correlacionada com a retratibilidade (BOWYER et al., 2003). Podem-se estimar as propriedades mecânicas da madeira por meio de equações de regressão em que a massa específica é a única variável independente (FOREST PRODUCTS LABORATORY, 2010; ARAÚJO, 2007; DIAS; LAHR, 2004).

A contração e a expansão higroscópica da madeira são dois dos mais importantes problemas práticos que ocorrem durante a utilização da madeira, como consequência da mudança do teor de umidade (OLIVEIRA; SILVA, 2003). A formação das tensões de secagem também está relacionada com os coeficientes de contração da madeira (KEEY et al., 2000).

As correlações entre a massa específica e as contrações radial e tangencial são consideravelmente menores que as observadas entre a primeira variável e as propriedades mecânicas. Modelos polinomiais, logarítmicos e exponenciais, quando ajustados para a contração radial ou tangencial média de um número representativo de espécies, não atingem coeficientes de determinação acima de $25 \%$. Os maiores coeficiente de determinação são encontrados quando são ajustados modelos de regressão entre a massa específica e o coeficiente de contração radial (ARAÚJO, 2007; DIAS; LAHR, 2004).

O objetivo deste trabalho foi determinar e comparar a massa específica aparente a $12 \%$ de umidade, a massa específica básica e os coeficientes de contração radial, tangencial e volumétricos da madeira de Eucalyptus grandis W. Hill ex Maiden, Corymbia torelliana F. Muell., Eucalyptus cloeziana F. Muell., Eucalyptus pilularis Sm., Eucalyptus resinifera Sm. e do híbrido Eucalyptus grandis W. Hill ex Maiden x Eucalyptus urophylla S. T. Blake.

\section{MATERIAL E MÉTODOS}

\section{Espécies e características do povoamento}

A madeira de Corymbia torelliana, Eucalyptus cloeziana, Eucalyptus resinifera e Eucalyptus pilularis foi obtida a partir de plantios experimentais com 17 anos, inicialmente implantados no espaçamento 2,5 x 3,0 m, no município de Garuva, SC. A madeira de Eucalyptus grandis é procedente de um plantio de 14 anos, implantado no espaçamento $2,5 \times 3,0 \mathrm{~m}$ em Piçarras, SC, e a do híbrido Eucalyptus grandis x Eucalyptus urophylla é proveniente de um plantio com oito anos, implantado no espaçamento 2,0 x 2,6 m em Barra Velha, SC.

\section{Massa específica básica e aparente}

A massa específica básica foi determinada como sendo a relação entre a massa anidra, obtida após estabilização em estufa a $103 \pm 2{ }^{\circ} \mathrm{C}$, e o volume saturado, determinado pelo método estereométrico.

A massa específica aparente foi determinada como sendo a relação entre a massa e o volume após a estabilização da massa dos corpos de provas mantidos em uma câmara climatizada em $20{ }^{\circ} \mathrm{C}$ e 65\% de umidade relativa. De acordo com as equações apresentadas por Forest Products Laboratory (2010), a umidade de equilíbrio nessas condições é de $12 \%$.

As duas variáveis foram determinadas em corpos de prova com as dimensões nominais de $25 \times 25 \times 100 \mathrm{~mm}$, retirados de cinco toras basais por espécie. O método A da norma ASTM D2395-07 foi utilizado para a determinação do volume.

\section{Coeficiente de contração radial, tangencial e volumétrica}

Os coeficientes de contração radial, tangencial e volumétrica foram determinados utilizando-se os mesmos corpos de prova utilizados para a determinação da massa específica. A contração total foi determinada como sendo a relação percentual entre a variação na dimensão observada entre o estado saturado e o anidro, em relação à dimensão saturada. O procedimento seguiu as recomendações da norma ASTM D143-09.

\section{Análise estatística}

As médias por espécie das três propriedades físicas analisadas foram comparadas pelo teste de Tukey $(\alpha=5 \%)$, e a relação entre massa específica básica e retratibilidade foi determinada através de coeficientes de correlação e do ajuste de equações lineares. 
A tabela 1 foi utilizada para classificar a massa específica aparente e os coeficientes de contração da madeira.

Tabela 1. Limites de classe para a massa específica aparente $\left(\rho_{12 \%}\right)$ e para o coeficiente de contração radial $\left(\beta_{\mathrm{r}}\right)$, tangencial $\left(\beta_{\mathrm{t}}\right)$ e volumétrica $\left(\beta_{\mathrm{v}}\right)$.

Table 1. Limits of classes for apparent specific gravity, radial, tangential and volumetric shrinkage.

\begin{tabular}{|c|c|c|c|c|c|}
\hline \multicolumn{2}{|c|}{$\rho_{12 \%}\left(\mathrm{~kg} / \mathrm{m}^{3}\right)$} & & $\beta_{\mathrm{r}}(\%)$ & $\beta_{\mathrm{t}}(\%)$ & $\beta_{\mathrm{v}}(\%)$ \\
\hline Muito leve & Até 400 & \multirow{2}{*}{ Baixo } & \multirow[b]{2}{*}{ Até 3,51 } & \multirow{2}{*}{ Até 7,43} & \multirow{2}{*}{ Até 12,32 } \\
\hline Leve & $400<x \leq 550$ & & & & \\
\hline $\begin{array}{l}\text { Moderadamente } \\
\text { pesada }\end{array}$ & $550<x \leq 750$ & \multirow[t]{2}{*}{ Médio } & \multirow[t]{2}{*}{3,52 a 5,59} & \multirow[t]{2}{*}{7,44 a 11,93} & \multirow[t]{2}{*}{12,33 a 19,39} \\
\hline Pesada & $750<x \leq 950$ & & & & \\
\hline Muito pesada & 950 ou acima & Alto & 5,60 ou acima & 11,94 ou acima & 19,40 ou acima \\
\hline
\end{tabular}

\section{RESULTADOS E DISCUSSÃO}

\section{Massa específica básica e aparente}

De acordo com a massa específica básica (Tabela 2), as espécies agrupam-se em quatro grupos distintos, sendo o primeiro formado pelo híbrido Eucalyptus grandis x Eucalyptus urophylla, apresentando a menor massa específica básica média, seguido das espécies Eucalyptus grandis e Eucalyptus pilularis, formando o segundo e o terceiro grupo, respectivamente. As três espécies restantes formam o quarto grupo, com os valores médios mais elevados.

A massa específica aparente média (Tabela 2) obtida para as cinco espécies e para o híbrido permitiu reuni-las em três grupos distintos estatisticamente. A diferença observada está restrita à madeira das espécies Eucalyptus grandis e Eucalyptus pilularis, diferentes estatisticamente na massa específica básica, mas que formam um único grupo na massa específica aparente.

Tabela 2. Número de peças $(\mathrm{N})$ utilizadas para obtenção da massa específica básica ( $\left.\rho_{\text {bás }}\right)$ e massa específica aparente $\left(\rho_{12 \%}\right)$ por espécie.

Table 2. Number of specimens $(\mathrm{N})$ utilized for determination of basic specific gravity ( $\left.\rho_{\text {bás }}\right)$ and aparent specific gravity $\left(\rho_{12 \%}\right)$ by specie.

\begin{tabular}{|c|c|c|c|c|}
\hline \multirow{2}{*}{ Espécie } & \multicolumn{2}{|c|}{$\rho_{\text {bás }}\left(\mathbf{k g} / \mathbf{m}^{3}\right)$} & \multicolumn{2}{|c|}{$\rho_{12 \%}\left(\mathrm{~kg} / \mathrm{m}^{3}\right)$} \\
\hline & $\mathbf{N}$ & Média & $\mathbf{N}$ & Média \\
\hline Corymbia torelliana & 97 & $\begin{array}{c}587,5 \mathrm{~d} \\
(62,9)\end{array}$ & 63 & $\begin{array}{c}616,2 \mathrm{c} \\
(51,0)\end{array}$ \\
\hline Eucalyptus cloeziana & 78 & $\begin{array}{c}614,0 \mathrm{~d} \\
(76,5)\end{array}$ & 43 & $\begin{array}{c}623,8 \mathrm{c} \\
(48,7)\end{array}$ \\
\hline Eucalyptus grandis & 57 & $\begin{array}{c}463,4 \mathrm{~b} \\
(50,9)\end{array}$ & 36 & $\begin{array}{c}501,9 \mathrm{~b} \\
(70,8)\end{array}$ \\
\hline Eucalyptus grandis x Eucalyptus urophylla & 78 & $\begin{array}{c}405,2 \mathrm{a} \\
(37,1)\end{array}$ & 35 & $\begin{array}{c}421,5 \mathrm{a} \\
(37,5)\end{array}$ \\
\hline Eucalyptus pilularis & 95 & $\begin{array}{c}506,4 \mathrm{c} \\
(69,3)\end{array}$ & 86 & $\begin{array}{c}517,5 \mathrm{~b} \\
(71,2)\end{array}$ \\
\hline Eucalyptus resinifera & 70 & $\begin{array}{c}585,9 \mathrm{~d} \\
(79,2)\end{array}$ & 52 & $\begin{array}{c}638,1 \mathrm{c} \\
(65,3)\end{array}$ \\
\hline
\end{tabular}

Médias seguidas na coluna pela mesma letra minúscula não diferem significativamente pelo teste de Tukey $(\alpha=5 \%)$. Valores entre parênteses abaixo das médias são os desvios padrão.

De acordo com os valores médios encontrados para massa específica aparente e segundo os limites apresentados na tabela 1, a madeira do híbrido Eucalyptus grandis x Eucalyptus urophylla, a de Eucalyptus grandis e a de Eucalyptus pilularis classifica-se como leve, e as demais espécies são classificadas como madeira moderadamente pesada. Como há relações entre a massa específica e as propriedades mecânicas, apresentadas por Forest Products Laboratory (2010), Araújo (2007) e Dias e Lahr (2004), entre outros, é de se esperar que as espécies classificadas como leves tenham limitações

FLORESTA, Curitiba, PR, v. 45, n. 2, p. 329 - 336, abr. / jun. 2015.

Eleotério, J. R. et al.

ISSN eletrônico 1982-4688 / ISSN impresso 0015-3826

DOI: $10.5380 /$ rf.v45i2.34699 
mais amplas que as demais, especialmente se a exigência envolver resistência mecânica, como no caso de madeira para embalagens, paletts e outros materiais logísticos.

Os coeficientes de variação médios de $11,75 \%$ e de $10,51 \%$ para a massa específica aparente e para a massa específica básica são próximos de $10 \%$, apresentados como coeficientes médios para a massa específica (FOREST PRODUCTS LABORATORY, 2010; BOWYER et al., 2003).

\section{Coeficiente de contração radial, tangencial e volumétrica}

Os grupos formados na comparação de médias dos valores de contração (Tabela 3) foram os mesmos, independentemente do plano analisado, se radial, tangencial ou volumétrico. Esse fato pode ser explicado pelo efeito aditivo da contração radial e tangencial na formação da contração volumétrica e pela correlação mediana $(36,6 \%)$ e significativa $\left(\mathrm{p}=1,19 * 10^{-5}\right)$ entre a contração radial e a tangencial.

Tabela 3. Número de peças $(\mathrm{N})$ utilizadas para determinação dos coeficientes médios de contração radial $(\beta r)$, tangencial $(\beta \mathrm{t})$ e volumétrica $(\beta \mathrm{v})$.

Table 3. Number of specimens $(\mathrm{N})$ utilized for determination of radial $(\beta \mathrm{r})$, tangencial $(\beta \mathrm{t})$ and volumetric $(\beta v)$ shrinkage coefficients.

\begin{tabular}{lcccc}
\hline Espécie & $\mathbf{N}$ & $\boldsymbol{\beta r}(\%)$ & $\boldsymbol{\beta t}(\%)$ & $\boldsymbol{\beta v}(\%)$ \\
\hline Corymbia torelliana & 31 & $5,5 \mathrm{bc}$ & $9,2 \mathrm{bc}$ & $14,2 \mathrm{bc}$ \\
& & $(1,4)$ & $(1,6)$ & $(1,6)$ \\
Eucalyptus cloeziana & 30 & $5,6 \mathrm{c}$ & $9,1 \mathrm{c}$ & $14,5 \mathrm{c}$ \\
& & $(0,7)$ & $(0,9)$ & $(1,2)$ \\
Eucalyptus grandis & 19 & $4,1 \mathrm{a}$ & $7,1 \mathrm{a}$ & $11,7 \mathrm{a}$ \\
& & $(1,2)$ & $(0,9)$ & $(1,5)$ \\
Eucalyptus grandis x Eucalyptus urophylla & 22 & $4,5 \mathrm{a}$ & $9,1 \mathrm{a}$ & $13,4 \mathrm{a}$ \\
& & $(0,9)$ & $(1,9)$ & $(2,4)$ \\
Eucalyptus pilularis & 22 & $4,7 \mathrm{ab}$ & $8,1 \mathrm{ab}$ & $12,2 \mathrm{ab}$ \\
& & $(1,4)$ & $(1,7)$ & $(2,7)$ \\
Eucalyptus resinifera & 12 & $6,0 \mathrm{c}$ & $10,7 \mathrm{c}$ & $16,3 \mathrm{c}$ \\
& & $(1,0)$ & $(1,5)$ & $(2,2)$ \\
\hline
\end{tabular}

Médias seguidas na coluna pela mesma letra minúscula não diferem significativamente pelo teste de Tukey $(\alpha=5 \%)$. Valores entre parênteses abaixo das médias são os desvios padrão.

A madeira das espécies Eucalyptus cloeziana e Eucalyptus resinifera apresentou os maiores coeficientes de contração, a espécie Eucalyptus grandis e o híbrido Eucalyptus grandis x Eucalyptus urophylla os menores e as outras duas espécies formaram um grupo intermediário, ligado aos grupos anteriores. Esses grupos não são exatamente os mesmos formados na comparação de médias da massa específica, indicando a independência parcial dessas duas propriedades físicas, apesar de a tendência de maior massa específica resultar em maior coeficiente de contração.

De acordo com os limites apresentados na tabela 1, são altos os coeficientes de contração radial de Eucalyptus cloeziana e Eucalyptus resinifera. Por outro lado, são baixos os coeficientes de contração tangencial e volumétrica de Eucalyptus grandis e volumétrica de Eucalyptus pilularis. Os demais podem ser classificados como médios.

As correlações entre a massa específica básica e o coeficiente de contração foi de $63,4 \%$ no plano radial, de $38,3 \%$ no plano tangencial e de $54,5 \%$ para a contração volumétrica. Todas essas correlações mostraram-se significativas a 1,0\% de probabilidade de erro.

$\mathrm{Na}$ tabela 4 são apresentadas as equações de regressão linear simples para os coeficientes de contração em função da massa específica básica. Os modelos apresentam coeficientes de determinação maiores que os apresentados por Araújo (2007) e por Dias e Lahr (2004), possivelmente por serem ajustados para espécies com características anatômicas e químicas mais próximas que as utilizadas pelos autores citados. Outra hipótese que explica o melhor ajuste é a utilização de valores individuais de massa específica básica e contração e não médias por espécie. Da mesma forma que observado pelos autores acima, o ajuste foi melhor no plano radial que no tangencial.

Apesar da significância dos modelos, observa-se nas figuras 1 a 3, pela dispersão dos dados ao redor do modelo, que a massa específica não é o único parâmetro que explica os coeficientes de 
contração. Possivelmente a organização microfibrilar, a acessibilidade aos sítios de adsorção, relacionada com a composição química, e a própria organização anatômica, definida pela forma e proporção dos diferentes elementos anatômicos, influenciam nessa propriedade mecânica. Lima et al. (2014) encontraram relação inversa entre a contração volumétrica e o diâmetro dos vasos em Eucalyptus grandis. Marques (2008) também encontrou essa relação para 41 espécies tropicais, além de relação inversa com a largura do lúmen das fibras e relação direta com a frequência de poros e a espessura da parede das fibras.

Tabela 4. Equações para estimar a contração em função da massa específica básica $\left(\mathrm{kg} / \mathrm{m}^{3}\right)$, com coeficiente de determinação ajustado $\left(\mathrm{R}_{\mathrm{aj}}{ }^{2}\right)$, erro padrão da estimativa $\left(\mathrm{S}_{\mathrm{yx}}\right)$, valor do teste $\mathrm{F}$ para regressão $(\mathrm{F})$ e probabilidade de erro do teste $\mathrm{F}(\mathrm{P})$.

Table 4. Equations to estimate the shrinkage coefficient as a function of basic specific gravity $\left(\mathrm{kg} / \mathrm{m}^{3}\right)$, with adjusted coefficient of determination $\left(\mathrm{R}_{\mathrm{aj}}{ }^{2}\right)$, standard error of the estimate $\left(\mathrm{S}_{\mathrm{yx}}\right), \mathrm{F}$ test value for the regression $(\mathrm{F})$ and $\mathrm{F}$ test probability of error $(\mathrm{P})$.

\begin{tabular}{lccccc}
\hline Contração & \multicolumn{1}{c}{ Equação } & $\mathbf{R}_{\mathbf{a j}}{ }^{2}$ & $\mathbf{S}_{\mathbf{y x}}$ & $\mathbf{F}$ & $\mathbf{P}$ \\
\hline Radial & $\beta_{\mathrm{r}}=0,0073 \rho_{\text {Bás }}+0,7492$ & 0,398 & 0,973 & 90,1 & $1,16^{*} 10^{-16}$ \\
\hline Tangencial & $\beta_{\mathrm{t}}=0,0060 \rho_{\text {Bás }}+5,2992$ & 0,140 & 1,58 & 23,0 & $4,23^{*} 10^{-6}$ \\
\hline Volumétrica & $\beta_{\mathrm{v}}=0,0117 \rho_{\text {Bás }}+6,7453$ & 0,292 & 1,96 & 56,6 & $6,88^{*} 10^{-12}$ \\
\hline
\end{tabular}

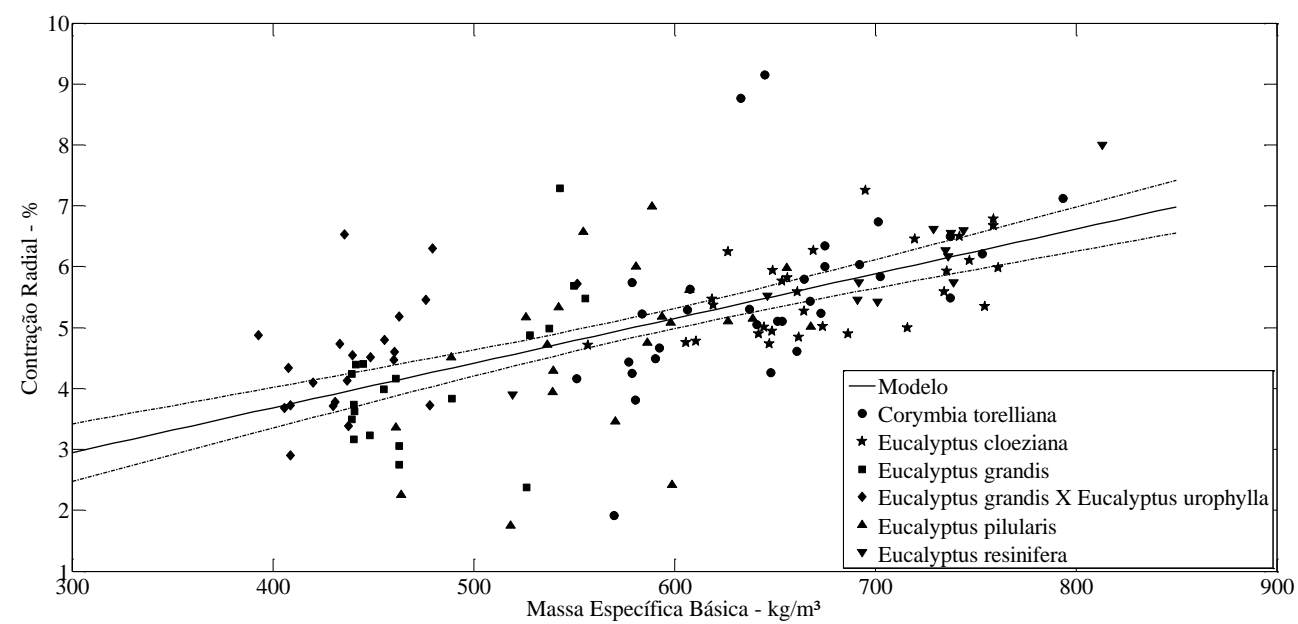

Figura 1. Relação entre contração radial e massa específica básica.

Figure 1. Relationship between radial shrinkage and basic specific gravity.

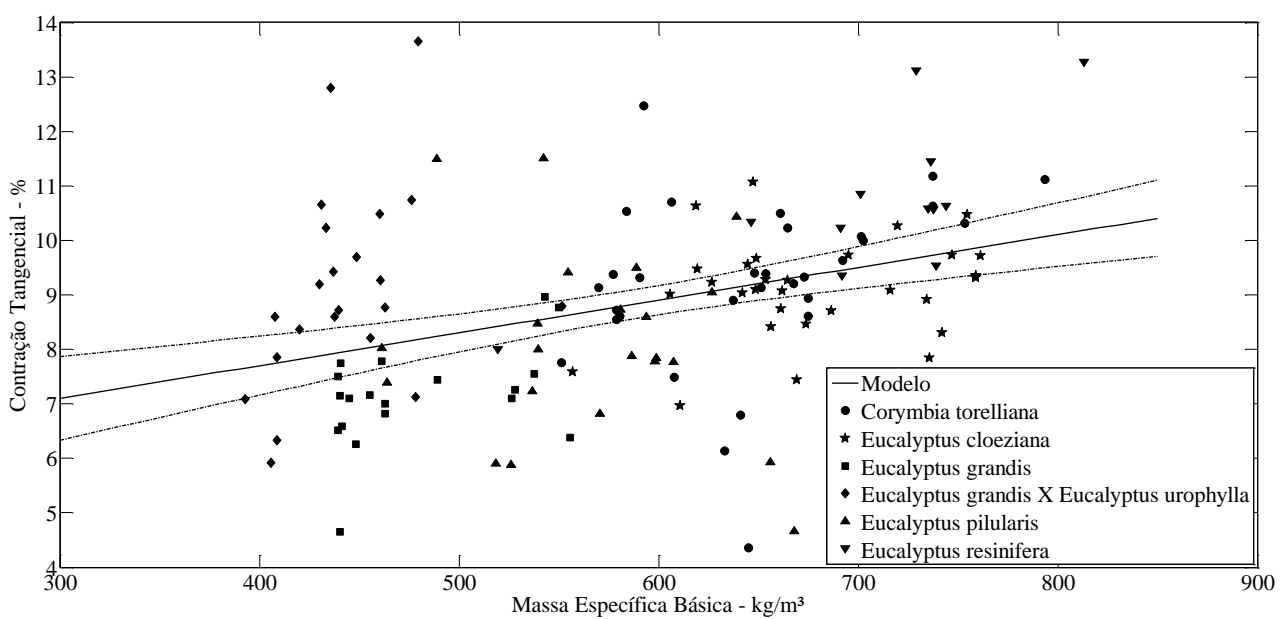

Figura 2. Relação entre contração tangencial e massa específica básica.

Figure 2. Relationship between tangential shrinkage and basic specific gravity.

FLORESTA, Curitiba, PR, v. 45, n. 2, p. 329 - 336, abr. / jun. 2015.

Eleotério, J. R. et al.

ISSN eletrônico 1982-4688 / ISSN impresso 0015-3826 


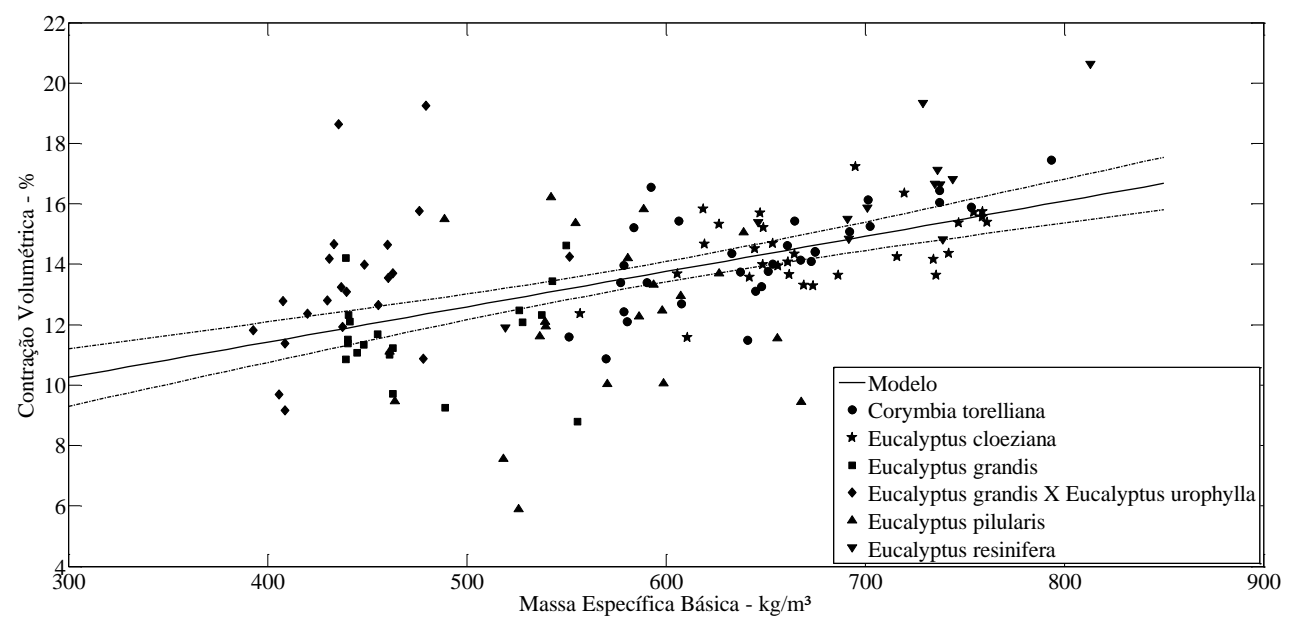

Figura 3. Relação entre contração volumétrica e massa específica básica.

Figure 3. Relationship between volumetric shrinkage and basic specific gravity.

Há necessidade de complementar os modelos com outras características da madeira, como tipo e teor de extrativos, ângulo microfibrilar, e proporção e disposição dos vários elementos anatômicos, entre outras características. Infelizmente, a determinação dessas propriedades não é tão simples e rápida como a determinação da massa específica básica.

A contração tangencial e volumétrica da madeira do híbrido Eucalyptus grandis x Eucalyptus urophylla, cujos valores desviam dos modelos, por exemplo, pode estar relacionada à idade das árvores, mais novas, com maior proporção de lenho juvenil e com menor deposição de extrativos formadores do cerne, entre outras características relacionadas à higroscopicidade e à anisotropia.

\section{CONCLUSÕES}

- A madeira mais leve é a formada pelo híbrido Eucalyptus grandis x Eucalyptus urophylla, que diferiu das demais tanto na massa específica básica como na aparente.

- A madeira do híbrido Eucalyptus grandis x Eucalyptus urophylla, de Eucalyptus grandis e de Eucalyptus pilularis classifica-se como leve.

- As espécies Corymbia torelliana, Eucalyptus cloeziana e Eucalyptus resinifera formam um grupo homogêneo tanto na massa específica básica como na aparente, cuja madeira pode ser classificada como moderadamente pesada.

- Independentemente da contração analisada, se radial, tangencial ou volumétrica, os grupos formados na comparação de médias foram os mesmos.

- Eucalyptus cloeziana e Eucalyptus resinifera apresentaram os maiores coeficientes de contração.

- $\quad$ Na madeira da espécie Eucalyptus grandis e do híbrido Eucalyptus grandis $x$ Eucalyptus urophylla foram observados os menores coeficientes de contração.

- Os coeficientes de contração radial de Eucalyptus cloeziana e Eucalyptus resinifera são altos. Já os coeficientes de contração tangencial e volumétrica de Eucalyptus grandis e volumétrica de Eucalyptus pilularis são baixos. Os demais classificam-se como médios.

- As correlações observadas, significativas, mas de média magnitude, confirmam que a massa específica não é o único parâmetro correlacionado com os coeficientes de contração, havendo a necessidade de complementar os modelos com outras características da madeira.

\section{REFERÊNCIAS}

AMERICAN SOCIETY FOR TESTING AND MATERIALS (ASTM). ASTM D143-09: Standard test methods for small clear specimens of timber. West Conshohocken: ASTM International, 2009. 
. ASTM D2395 - 14: Standard test methods for density and specific gravity (relative density) of wood and wood-based materials. West Conshohocken: ASTM International, 2009.

ARAÚJO, H. J. B. de. Relações funcionais entre propriedades físicas e mecânicas de madeiras tropicais brasileiras. Floresta, Curitiba, v. 37, n. 3, p. 399 - 416, 2007.

BOWYER, J. L.; SHMULSKY, R.; HAYGREEN, J. G. Forest products and wood science: an introduction. Ames Iowa : Iowa State Press, 2003. 4th ed. xiv, 554 p.

DIAS, F. M.; LAHR, F. A. R. Estimativa de propriedades de resistência e rigidez da madeira através da densidade aparente. ScientiaForestalis, Piracicaba, n. 65, p. 102 - 113, 2004.

FOREST PRODUCTS LABORATORY. Wood Handbook: Wood as an engineering material. Madison, WI: U.S. Department of Agriculture, Forest Service, Forest Products Laboratory, 2010. 509 p. (General Technical Report, FPL-GTR-190).

KEEY, R. B.; LANGRISH, T. A. G.; WALKER, J. C. F. Kiln-drying of lumber. Berlim: Springer Verlag, 2000. xi, $326 \mathrm{p}$.

LIMA, I. L.; LONGHI, E. L.; FREITAS, M. L. M.; ZANATTO, A. C. S.; ZANATA, M.; FLORSHEIM, S. M. B.; BORTOLETTO JÚNIOR., G. Physical-mechanical and anatomical characterization in 26-yearold Eucalyptus resinifera wood. Floresta e Ambiente, Seropédica, v. 1, n. 21, p. 91 - 98, jan. 2014. Disponível em: <http://www.scielo.br/pdf/floram/v21n1/aop_floram_055513.pdf>. Acesso em: 07 ago. 2014.

MARQUES, M. H. B. Agrupamento de 41 espécies de madeiras da Amazônia para secagem baseado em características anatômicas e físicas. 2008. 141 f. Tese (Doutorado) - Universidade de Brasília, Brasília, 2008.

OLIVEIRA, J. T. da S.; SILVA, J. de C. Variação radial da retratibilidade e densidade básica da madeira de Eucalyptus saligna Sm. Revista Árvore, Viçosa, v. 27, n. 3, p. 381 - 385, 2003. 
FLORESTA, Curitiba, PR, v. 45, n. 2, p. 329 - 336, abr. / jun. 2015. Eleotério, J. R. et al. 